Revised, accepted manuscript

Appeared in Fluid Dynamics Research, 47 (3), Seiten 1-17.

DOI: 10.1088/0169-5983/47/3/035508.

ISSN 0169-5983.

\title{
Excitation of vortex meandering in shear flow
}

\author{
Josef Schröttle $\ddagger$, Andreas Dörnbrack $\S$ and Ulrich Schumann|| \\ Deutsches Zentrum für Luft- und Raumfahrt, Institut für Physik der Atmosphäre, \\ Oberpfaffenhofen, Germany \\ E-mail: josef.schroettle@dlr.de
}

\begin{abstract}
.
This paper investigates the evolution of a streamwise aligned columnar vortex with vorticity $\boldsymbol{\omega}$ in axial background shear of magnitude $\Omega$ by means of linear stability analysis and numerical simulations. A long wave mode of vorticity normal to the plane spanned by the background shear vector $\boldsymbol{\Omega}$ and the vorticity of the vortex is excited by an instability. Stationary wave modes of vertical and lateral vorticity amplify. In order to form a helical vortex, the lateral and vertical vorticity can be phase shifted by half a wave length. The linear and nonlinear evolutions of the vortex in the shear flow are studied numerically. Linearized simulations confirm the results of the stability analysis. The nonlinear simulations reveal the further evolution of the helix in the shear flow. The linearly excited mode persists in co-existence with evolving smaller scale instabilities until the flow becomes fully turbulent at a time of $\mathcal{O}\left(100 \Omega^{-1}\right)$. Turbulent mixing damps the amplifying mode. The described phenomenon of vortex meandering may serve as alternative explanation for the excitation of wind turbine wake meandering in the atmospheric boundary layer.
\end{abstract}

Keywords: Atmospheric boundary layer, wind energy, wake vortex, meandering

$\ddagger$ Corresponding author: josef.schroettle@dlr.de

$\S$ andreas.doernbrack@dlr.de

|| ulrich.schumann@dlr.de 


\section{Introduction}

In the Earth's atmosphere vortices occur over a broad range of scales and scenarios. Tornadoes, dust devils, or wake vortices behind aircraft and wind turbines are only a few examples. The atmospheric boundary layer (ABL) is modified by the flow through and around a wind turbine. The upstream region in front of a wind turbine defines the inflow. Downstream, the rotating turbine generates a wake due to its drag. The wake is characterized by a velocity deficit. In addition, a lift force acts at the rotating blades and tip vortices are shed. In the near wake, these tip vortices interact, merge, and eventually diffuse. Okulov \& Sorensen (2007) constituted a conceptual model for the diffusional merging of tip vortices. In a downstream distance of about three rotor diameters the tip vortices have diffused (Ivanell et al., 2010). There, the far-wake region begins and the wake forms one vortex. The mean flow advects this vortex downstream and a coherent columnar vortex evolves. In their conceptual model Okulov \& Sorensen (2007) applied a Rankine vortex to model this far-wake vortex. Prandtl and Betz modeled the turbine wake as a rigidly rotating vortex with uniform vorticity (Sørensen, 2011). Käsler (2011) observed the far-wake vortex behind a rotating wind turbine in the ABL. These observations indicate that the far-wake vortex can be modeled with a smooth vorticity distribution as e.g. a Lamb-Oseen vortex.

In the ABL the far-wake vortex does not propagate downstream in a straight line. The far wake is often unsteady and the wake forms so called meanders. Originally, a meander refers to a winding river. Behind a wind turbine, meandering depicts the transverse sinusoidal oscillations of the wake. Wake meandering behind a wind turbine was observed in the ABL, e.g. by Hirth et al. (2012) by means of radar measurements or with lidar observations by e.g. Trujillo et al. (2011). In wind and water tunnel experiments meandering of turbine wakes was observed by several investigators (Medici \& Alfredsson, 2006; Chamorro \& Porté-Agel, 2010; Iungo et al., 2013; Okulov et al., 2014). There, the blades of the model turbines rotated with varying characteristic tip speed ratio and extracted power from the mean flow. In the laboratory experiments by Iungo et al. (2013) the mean inflow velocity was constant with height across the turbine diameter. Based on measured velocity profiles from these wind tunnel experiments, Iungo et al. (2013) studied the instability of a wind turbine wake by means of linear stability analysis.

The origins of the wake meandering phenomenon are still under debate (Okulov et al., 2014). Larsen et al. (2008) developed and successfully applied an empirical model for the prediction of the wake meandering. Thereby, Larsen et al. (2008) considered the wake as a passive tracer. The assumption for their model is the following: coherent structures in the form of upstream vortices larger than the turbine diameter move downstream with a constant speed and advect the wake in transverse direction. This advection forms the meandering wake. Trujillo et al. (2011) quantitatively compared the empirical model prediction with lidar measurements of the unsteady wake meandering. From this comparison Trujillo et al. (2011) concluded that modeling the wake as a 
passive tracer is a fair approximation. Medici \& Alfredsson (2006) followed another hypothesis and state vortex shedding (as behind a bluff body) by the rotating wind turbine as a cause for the wake meandering. Okulov et al. (2014) found meandering in the form of a low-frequency oscillation of the far-wake velocity field in laboratory experiments. In these experiments no large eddies existed in the upstream flow and the mean inflow velocity was uniform with height across the rotor diameter.

So far, the effect of a strong background shear in the inflow velocity profile on the far-wake vortex has not been investigated. For example, in the nocturnal boundary layer, vertical shear magnitudes up to $\Omega=0.1 \mathrm{~s}^{-1}$ occur during low-level jet events, as observed by Käsler et al. (2010), at the typical height of the wind turbine (Banta et al., 2013). These large shear values are comparable to or exceed the vorticity of the far-wake vortex. Käsler (2011) referred to the vortical flow of the far-wake vortex as swirl. The goal of this paper is to study the excitation of far-wake meandering as an intrinsic instability of this vortex in strong shear flow.

The following questions shall be addressed:

(i) Can strong background shear excite meandering of the far-wake vortex?

(ii) What is the direction and frequency of the excited modes?

(iii) Does the excited mode persist as the flow evolves?

Our paper is organized as follows: In section 2, we present a linear stability analysis of a columnar vortex in strong background shear. Numerical simulations of a perturbed single columnar Lamb-Oseen vortex in strong background shear complement the linear stability analysis. The corresponding results of the LESs are presented in section 3 . Finally, we discuss our results in section 4 and conclude in section 5 .

\section{Linear theory}

\subsection{Conceptual model}

A swirl flow in the wake of a rotating wind turbine exists if power is extracted from the mean flow. A short distance downstream of the turbine, the vorticity originating from the tip vortices merges by turbulent mixing into one vortex with mean vorticity $\boldsymbol{\omega}$. As the turbine extracts energy from the mean flow, a velocity deficit $\Delta U$ forms in its wake. In the far wake, $\boldsymbol{\omega}$ and $\Delta U$ decay because of turbulent mixing between the vortex and ambient air.

Glauert (1926) modeled this swirl flow with the actuator disk model based on momentum theory. In this model the far-wake vortex has uniform vorticity $\boldsymbol{\omega}$ across the turbine diameter $D \approx 100 \mathrm{~m}-200 \mathrm{~m}$. Laboratory experiments by Medici \& Alfredsson (2006) showed that the vortex core can be approximated by rigid body rotation at a downstream distance of about 3 rotor diameters; c.f. figure 16.b by Okulov \& Sorensen (2007). The decrease of $\Delta U$ in the far wake is seen, e.g. in figure 3 by Käsler et al. (2010) or figure 4 by Hirth et al. (2012). 
In the numerical simulations, we approximate the vortical flow in the far wake by a Lamb-Oseen vortex as defined by Saffman (1992). This representation is supported by observations of the swirl in the ABL. Figure 6.19 by Käsler (2011) shows a smooth vertical profile of the vortex velocity. From this vertical profile, we estimate the far-wake vorticity $\omega_{0} \lesssim \Omega / 2$. There, the vertical shear $\Omega=d U / d z$ is estimated from the wind lidar observations and has the same magnitude as the $\Omega$-values given by Banta et al. (2013).

For the linear stability analysis, we use an axially symmetric columnar vortex with radial velocity $V_{r}=0$. The vortex is aligned in streamwise $x$-direction and centered at $y=0$ and $z=0$ in a Cartesian coordinate system $\mathbf{x}=(x, y, z)$. The azimuthal velocity of the wake vortex $v_{\theta}=\omega_{0} r / 2$ at the position of the wind turbine is additionally calculated by the actuator disk approach as described by Meyers \& Meneveau (2013). Here, $\omega_{0}$ is the radially constant vorticity magnitude of the rigid wake vortex and $r=\sqrt{y^{2}+z^{2}}$. The shear induced by the velocity deficit $\Delta U / D$ can be estimated from observations by e.g. Trujillo et al. (2011); c.f. their figure 4. There, the shear at e.g. 4 rotor diameters downstream is small compared to the $\Omega$-values. Thus, we set $\Delta U=0$ in our conceptual model. The lateral and vertical wind components are set to $V=W=0$. The wind exhibits high velocity magnitudes in the shear flow and thus, we consider a neutrally stratified ABL.

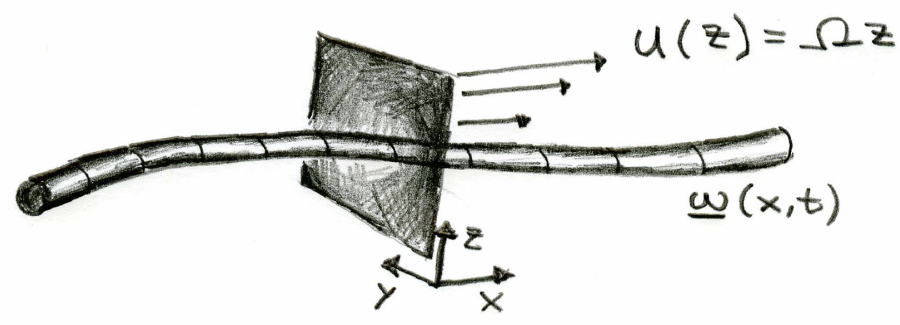

Figure 1. Configuration of the columnar vortex $\boldsymbol{\omega}$ in Cartesian coordinates $\mathbf{x}=$ $(x, y, z)$ with streamwise shear flow $U(z)=z \Omega$. The columnar vortex is perturbed with a long streamwise wave mode. The shaded textured rectangle indicates the $y z$ plane perpendicular to the columnar vortex.

\subsection{Vorticity equation, initial \& boundary conditions}

In this section we study the linear evolution of temporal and streamwise vorticity perturbations on a rigid vortex in strong axial shear flow as shown in figure 1 . The basis of our stability analysis is the prognostic equation for the vorticity vector

$$
\partial_{t} \widetilde{\boldsymbol{\omega}}=-(\widetilde{\mathbf{v}} \cdot \nabla) \widetilde{\boldsymbol{\omega}}+(\widetilde{\boldsymbol{\omega}} \cdot \nabla) \widetilde{\mathbf{v}}+\nu_{e d d y} \Delta \widetilde{\boldsymbol{\omega}}
$$

Thereby, $\partial_{t}$ is the Eulerian time derivate $\partial / \partial t$ and $\widetilde{\mathbf{v}}$ is the velocity vector. The symbols $\nabla$ and $\Delta$ have the usual meanings. We consider momentum diffusion with an effective eddy viscosity $\nu_{e d d y}$. 
In order to linearize equation (1), we decompose the total vorticity $\widetilde{\boldsymbol{\omega}}$ :

$$
\widetilde{\omega}=\underbrace{\Omega}_{\text {shear }}+\underbrace{\omega}_{\text {vortex vorticity }}
$$

in the background shear

$$
\Omega=\left(\begin{array}{c}
0 \\
\Omega \\
0
\end{array}\right)
$$

and the vorticity of the far-wake vortex

$$
\boldsymbol{\omega}(x, y, z, t)=\left(\begin{array}{c}
\omega_{x} \\
\omega_{y} \\
\omega_{z}
\end{array}\right) .
$$

Similarly, the total velocity field $\widetilde{\mathbf{v}}$ is decomposed in the following way:

$$
\widetilde{\mathbf{v}}=\underbrace{\mathbf{V}}_{\text {shear flow }}+\underbrace{\mathbf{v}}_{\text {vortex velocity }},
$$

whereby the background shear flow is given as plane parallel shear as follows

$$
\mathbf{V}(z)=\left(\begin{array}{c}
U \\
V \\
W
\end{array}\right)=\left(\begin{array}{c}
z \Omega \\
0 \\
0
\end{array}\right)
$$

The swirl flow of the vortex is described as

$$
\mathbf{v}(x, y, z, t)=\frac{1}{2} \omega_{0}(x, t)\left(\begin{array}{c}
0 \\
-z \\
y
\end{array}\right)+\left(\begin{array}{c}
0 \\
v^{\prime}(x, t) \\
w^{\prime}(x, t)
\end{array}\right) .
$$

Thereby, $\omega_{0}$ is the vortex vorticity. Inside the rigid vortex $\omega_{0}>0$. Outside of the rigid vortex, where $r=\sqrt{y^{2}+z^{2}}>D / 2$, the vortex vorticity $\omega_{0}=0$. The vortex is perturbed with a lateral and vertical velocity disturbance $v^{\prime}(x, t)$ and $w^{\prime}(x, t)$. The velocity field of the vortex and the perturbations satisfiy continuity equation $\nabla \cdot \mathbf{v}=0$.

\subsection{Linear approximation}

We linearize the vorticity equation (1) for the given background shear $\boldsymbol{\Omega}$ and vortical flow $\mathbf{v}$. We assume the vorticity magnitude of the rigid vortex $\omega_{0}$ to be small compared to the magnitude of the background shear $\Omega$. Due to the constant background shear in space and time the following prognostic equation for the vorticity $\boldsymbol{\omega}$ of the columnar vortex can be derived:

$$
\partial_{t} \boldsymbol{\omega}+\underbrace{(\widetilde{\mathbf{v}} \cdot \nabla) \boldsymbol{\omega}}_{\text {advection }}-\underbrace{(\widetilde{\boldsymbol{\omega}} \cdot \nabla) \widetilde{\mathbf{v}}}_{\text {tilting \& twisting }}-\underbrace{\nu_{\text {eddy }} \Delta \boldsymbol{\omega}}_{\text {diffusion }}=\mathbf{0} .
$$


The terms in equation (8) describe the advection, tilting, twisting, and diffusion of the wake vortex under the influence of background shear which is incorporated in the quantities $\widetilde{\boldsymbol{\omega}}$ and $\widetilde{\mathbf{v}}$. According to the definition of the background flow $\mathbf{V}$ in equation (6), the linearized streamwise advection reduces to

$$
\mathcal{A}_{\mathbf{V}}=(\mathbf{V} \cdot \nabla) \boldsymbol{\omega} .
$$

There, we neglect the term of second-order magnitude $(\mathbf{v} \cdot \nabla) \boldsymbol{\omega}$. The linearized tilting and twisting terms change the vorticity as follows:

$$
\mathcal{M}_{\Omega}=-(\boldsymbol{\omega} \cdot \nabla) \mathbf{V}-(\boldsymbol{\Omega} \cdot \nabla) \mathbf{v}=-\left(\begin{array}{c}
\omega_{z} \Omega \\
0 \\
\omega_{x} \Omega / 2
\end{array}\right) .
$$

The derived $\mathcal{M}_{\Omega}$ for this particular flow configuration contains only the terms $(\boldsymbol{\omega} \cdot \nabla) \mathbf{V}$ and $(\boldsymbol{\Omega} \cdot \nabla) \mathbf{v}$. The first term describes the tilting and twisting of the background shear flow by the vortex, whereas the second term describes the tilting and twisting of the vortical flow by the background shear. The term of second-order magnitude $(\boldsymbol{\omega} \cdot \nabla) \mathbf{v}$ is not part of the linear equation system. The term $(\boldsymbol{\Omega} \cdot \nabla) \mathbf{V}$ vanishes as the background flow only depends on $z$-direction. The result on the right hand side of equation (10) follows by substitution of the definitions from equations (6) and (7) for background velocity and vortex velocity. The evolving $\omega_{z}$ may induce oscillations in the transverse direction under time-dependent conditions, i.e. wake vortex meandering. Therefore, we denote the term $\mathcal{M}_{\Omega}$ as meandering.

The diffusion of vortex vorticity is given by

$$
\mathcal{D}_{\omega}=-\nu_{e d d y} \Delta \omega .
$$

Using the above abbreviations, equation (8) reads as follows:

$$
\partial_{t} \boldsymbol{\omega}+\underbrace{\mathcal{A}_{\mathbf{V}}}_{\text {advection }}+\underbrace{\mathcal{M}_{\Omega}}_{\text {meandering }}+\underbrace{\mathcal{D}_{\boldsymbol{\omega}}}_{\text {diffusion }}=\mathbf{0} \text {. }
$$

\subsection{Temporal stability analysis for rigid vortex in shear flow}

To study long wave modes in streamwise direction evolving over time, the ansatz for vorticity fluctuations are Fourier modes of the form

$$
\left(\begin{array}{l}
\omega_{x} \\
\omega_{y} \\
\omega_{z}
\end{array}\right)=\left(\begin{array}{c}
\widehat{\omega}_{x} \\
\widehat{\omega}_{y} \\
\widehat{\omega}_{z}
\end{array}\right) \exp [i(\alpha t+k x)]+c . c .,
$$

where $\left(\widehat{\omega}_{x}, \widehat{\omega}_{y}, \widehat{\omega}_{z}\right)$ are the amplitudes of the vorticity fluctuations, $k$ is the wave number in streamwise direction, $\alpha$ denotes the frequency of the waves, and c.c. is the complex conjugate. We use the common approximation of periodicity in $x$-direction. Hence, we assume small variations of the vortex vorticity in $x$-direction. The amplitudes $\widehat{\omega}_{x}$, $\widehat{\omega}_{y}, \widehat{\omega}_{z}$ and the frequency $\alpha$ may be complex. The wave number $k$ is real. Different combinations of perturbation amplitudes $\widehat{\omega}_{x}, \widehat{\omega}_{y}$, and $\widehat{\omega}_{z}$ are allowed. Modes where $\widehat{\omega}_{z}$ 
and $\widehat{\omega}_{y}$ differ by a factor of $i$ have phase shifted lateral and vertical vorticity components. The phase shift occurs in streamwise direction. This phase shift characterizes the corresponding solutions as a helix.

Here, ansatz (13) is substituted in the linearized vorticity equation (12) to obtain a linear system of algebraic equations for the deflection of the vortex under the influence of advection, diffusion, and meandering. The algebraic equation system for the evolution of the wave is

$$
(\alpha+\underbrace{U k}_{\text {advection }}-\underbrace{i \nu_{\text {eddy }} k^{2}}_{\text {diffusion }})\left(\begin{array}{c}
\widehat{\omega}_{x} \\
\widehat{\omega}_{y} \\
\widehat{\omega}_{z}
\end{array}\right)+i \underbrace{\left(\begin{array}{c}
\widehat{\omega}_{z} \Omega \\
0 \\
\widehat{\omega}_{x} \Omega / 2
\end{array}\right)}_{\text {meandering }}=\mathbf{0 .}
$$

We formulate the equation system in matrix form with $\gamma \equiv \alpha+U k-i \nu_{\text {eddy }} k^{2}$ as

$$
\underbrace{\left(\begin{array}{ccc}
\gamma & 0 & i \Omega \\
0 & \gamma & 0 \\
i \Omega / 2 & 0 & \gamma
\end{array}\right)}_{M}\left(\begin{array}{l}
\widehat{\omega}_{x} \\
\widehat{\omega}_{y} \\
\widehat{\omega}_{z}
\end{array}\right)=\mathbf{0}
$$

This system has a non-trivial solution, only if the determinant of the matrix $\|M\|=0$. The determinant $\|M\|=\gamma\left(\gamma^{2}+\Omega^{2} / 2\right)$. This cubic equation gives the eigenvalues of the matrix $M$ as $\gamma_{0}=0, \gamma_{1}=i \Omega / \sqrt{2}$, and $\gamma_{2}=-i \Omega / \sqrt{2}$, respectively. The background shear $\Omega$ is the eigenvector for eigenvalues $\gamma_{1}$ and $\gamma_{2}$.

\subsection{Dispersion relations}

Using the definition of $\gamma$ the following dispersion relations can be derived and are given as follows:

$$
\begin{aligned}
& \alpha_{0}=-\underbrace{U k}_{\text {advection }}+i \underbrace{\nu_{\text {eddy }} k^{2}}_{\text {diffusion }}, \\
& \alpha_{1}=-\underbrace{U k}_{\text {advection }}+i \underbrace{\nu_{\text {eddy }} k^{2}}_{\text {diffusion }}+i \underbrace{\Omega / \sqrt{2}}_{\text {meandering }}, \\
& \alpha_{2}=-\underbrace{U k}_{\text {advection }}+i \underbrace{\nu_{\text {eddy }} k^{2}}_{\text {diffusion }}-i \underbrace{\Omega / \sqrt{2}}_{\text {meandering }} .
\end{aligned}
$$

Neutrally stable oscillations only occur if the meandering frequencies $\alpha_{0}, \alpha_{1}$, and $\alpha_{2}$ are real. Here, all eigenvalues are composed of real and imaginary numbers. The real numbers contain terms due to advection. One imaginary part of the eigenfrequencies is due to diffusion and damps the solution. In the second and third eigenvalues $\Omega$ constitutes a time scale for the meandering as imaginary term. Dispersion relation (17) 
predicts amplifying unstable modes for shear values $\Omega>0$. The advection term is proportional to $k$, the diffusion is proportional to $k^{2}$, and the shear induced meandering is independent of wave number. The smaller the wave number and the longer the wave, the more pronounced becomes the meandering compared to diffusion and advection.

\subsection{Propagation of meandering waves}

To determine propagation velocities of the meandering waves, the respective phase speed $c_{p}$ and group velocity $c_{g}$ of the modes in streamwise direction are calculated (Vallis, 2006):

$$
c_{p}=k^{-1} \operatorname{Re}\left(\alpha_{1,2}\right)=-U \text {. }
$$

The phase speed has the same magnitude as the streamwise velocity $U$ and points in opposite direction. The propagation of the meandering wave is determined by the group velocity $c_{g}$ which is the partial derivative of the real part of $\alpha_{1,2}$ with respect to the wave number $k$ :

$$
c_{g}=\partial_{k} \operatorname{Re}\left(\alpha_{1,2}\right)=-U \text {. }
$$

The waves propagate against the mean flow $\mathbf{V}$ and move upstream in $x$-direction with a

velocity magnitude which equals the streamwise velocity $U$. Overall, the group velocity $c_{g}$ cancels with the advection velocity of the background flow and for a stationary observer, the meandering is a stationary wave. The group velocity equals the phase velocity and thus the waves are nondispersive.

\section{Large-eddy simulations}

To simulate the columnar vortex in strong shear, we conduct numerical simulations with the multiscale geophysical flow solver EULAG (Prusa et al., 2008). Here, we only employ a small portion of the full capabilities of the numerical model. The governing equations of fluid motion are cast in stationary Cartesian coordinates:

$$
\begin{aligned}
& \nabla \cdot \widetilde{\mathbf{v}}=0, \\
& D \widetilde{\mathbf{v}} / D t=-\nabla \widetilde{\pi}+\mathcal{D}^{\mathbf{v}}, \\
& D \widetilde{e} / D t=\mathcal{S}(\widetilde{e}),
\end{aligned}
$$

where $\tilde{\pi}$ is the pressure perturbation with respect to the undisturbed background profile normalized with constant density. $\mathcal{D}^{\mathbf{v}}$ is related to the deviatoric stress tensor as defined by Margolin et al. (1999) and represents turbulent mixing. The quantity $\widetilde{e}$ is the subgrid scale (SGS) turbulent kinetic energy and $\mathcal{S}(\widetilde{e})$ represents the respective production and dissipation terms (Margolin et al., 1999). The eddy viscosity coefficients are determined with the SGS closure model by Schumann (1975). Over the whole domain the effective average eddy viscosity in these simulations is approximately $10^{-3} \Omega D^{2}$. Sensitivity studies without explicit eddy viscosity are conducted using the implicit LES technique (Grinstein et al., 2007). For additional studies, the advection in equation (21) 


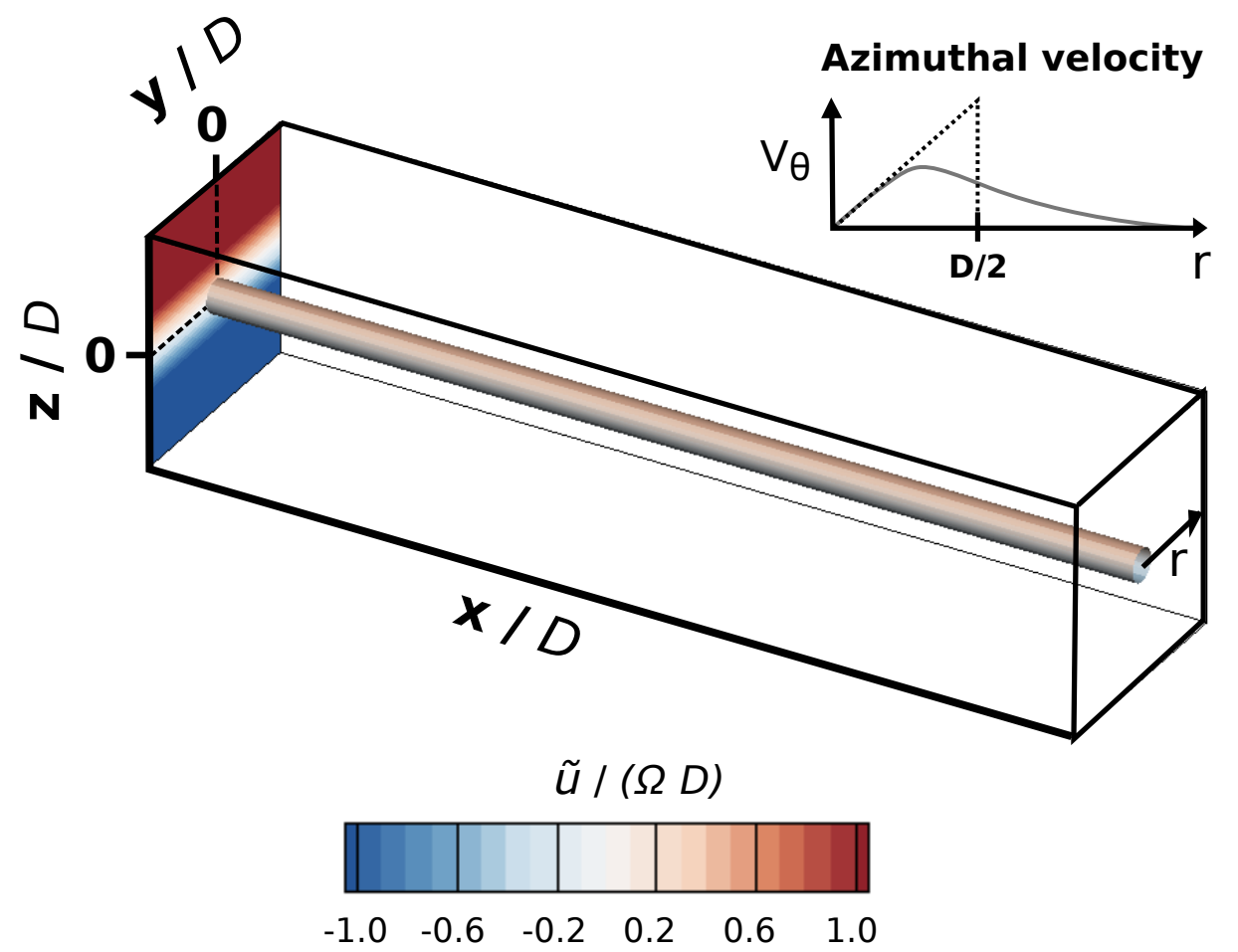

Figure 2. Configuration: a shear in the streamwise velocity $\widetilde{u}(z)$ is portrayed on the $y z$-plane. A vorticity isosurface $\omega_{x}=\omega_{0}$ denotes the streamwise aligned vortex. (shaded grey). In the upper right corner a sketch shows the azimuthal velocity profile $v_{\theta}$ of Lamb-Oseen vortex (grey line) and rigid vortex (dotted black line).

is linearized with respect to background shear flow $\mathbf{V}$. Thereby, a linear forcing due to shear $\Omega$ advects momentum numerically similar as the Lorentz force does in the magnetohydrodynamic equations by Smolarkiewicz \& Charbonneau (2013). A detailed description of the computational methods to solve the fully nonlinear governing equations numerically is given e.g. by Smolarkiewicz et al. (2014).

\subsection{Numerical setup}

The numerical domain is presented in figure 2. The domain has periodic boundaries in streamwise $x$-direction of length $L_{x}=10.3 D$ and Neumann boundary conditions for the velocity components in lateral $y$ - and vertical $z$-direction of lengths $L_{y}=L_{z}=2.6 \mathrm{D}$, respectively. The initial streamwise velocity $\widetilde{u}$ exhibits the linear shear $d U / d z=\Omega$ as depicted in the $y z$-plane in figure 2. This background shear is homogeneous across the numerical domain. Lateral and vertical velocity components are calculated from the azimuthal velocity of the Lamb-Oseen vortex:

$$
v_{\theta}(r)=\frac{\Gamma}{2 \pi r}\left(1-\exp \left(-\frac{r^{2}}{r_{c}^{2}}\right)\right),
$$

where $r_{c} \equiv D / 6$ is the vortex core radius at time $t_{0}$. Saffman (1992) defined the time dependent vortex core radius as $r_{c} \sim \sqrt{\nu t}$, where $\nu$ is the viscosity and in our case the 
eddy viscosity $\nu_{e d d y}$ in the ABL. $\Gamma$ is the vortex circulation $\Gamma=\int_{S} \boldsymbol{\omega} d S=\omega_{0} D^{2} \pi / 4$, where $S$ is the plane perpendicular to the vortex axis. The maximum tangential velocity of the vortex occurs at $r=D / 4$ and is $V_{\theta}=\omega_{o} D / 4$. First, the vertical position of the vortex is initially perturbed harmonically with a long streamwise wave mode $\lambda$ of amplitude $a$. Secondly, random numbers of small amplitude are superimposed on the initial velocity field and the vortex is centered with displacement amplitude $a=0$. A set of random numbers is applied, where the numbers are distributed in three dimensions and vary in $x-, y$-, and $z$-direction. Thirdly, the numbers are distributed consistently in lateral and vertical direction and only vary in $x$-direction. In additional studies, we vary the magnitude of $\omega_{0}$ up to a value of $\Omega$. According to the assumption of the linear stability analysis, we set $\omega_{0} \equiv \Omega / 10$ in the fully nonlinear simulation presented here. This corresponds to a turnover time of the vortex at a time scale of $\approx 10 \Omega^{-1}$. In the following, all variables are non-dimensionalized with vortex diameter $D$ and the background shear magnitude $\Omega$.

\subsection{Excitation of vortex meandering}

To visualize the dynamics in three dimensions and to identify the evolution of the vortex cores we use the $\lambda_{2}$-method. Thereby, $\lambda_{2}$ is the second eigenvalue of a tensor that identifies regions of high rotation and strain in the flow (Jeong et al., 1997). The eigenvalues of this tensor are $\lambda_{1} \geq \lambda_{2} \geq \lambda_{3}$. Jeong et al. (1997) find vortex cores for varying flow configurations at locations where two eigenvalues of the tensor are negative, i.e. $\lambda_{2}<0$. Our visualizations in figure 3 show the early evolution of the vortex with $\lambda_{2}$-contours. The contours represent the vortex cores with radius $r_{c}$ and are thinner than the vortex which carries vorticity across the vortex diameter. The vortex cores indicate the position of the vortex center line. The initially streamwise aligned vortex undergoes

several stages. At the early stage the vortex gradually undulates with small amplitude $\approx a<D$ in $y$-direction with a long wave of length $\lambda \gg D$ in streamwise $x$-direction. This wave is phase shifted to the initial mode in $z$-direction by $\lambda / 4$ during the excitation of the vortex meandering. This excitation occurs continuously throughout the depicted dimensionless times $8 \Omega^{-1}, 10 \Omega^{-1}$, and $12 \Omega^{-1}$.

\subsection{Transition to nonlinear flow}

Figure 4 shows the temporal evolution of the simulated streamwise velocity component $\widetilde{u}$ in an $y z$-plane at $x=L_{x} / 2$ for subsequent times. There, the vortical motion transports horizontal momentum up- and downwards around the vortex center. This deforms the shear at the early stage of the instability $\left(t \lesssim 12 \Omega^{-1}\right)$. Later, streamwise momentum of higher magnitude is transported inwards towards the vortex center after $16 \Omega^{-1}$. A consequential overturning of the shear is simulated at $t \approx 24 \Omega^{-1}$ leading to localized regions of $\partial_{z} \widetilde{u}<0$.

The deformation of the shear in the $y z$-plane has an effect on the temporal streamwise evolution of the columnar vortex as shown in figure 5. There, the temporal 

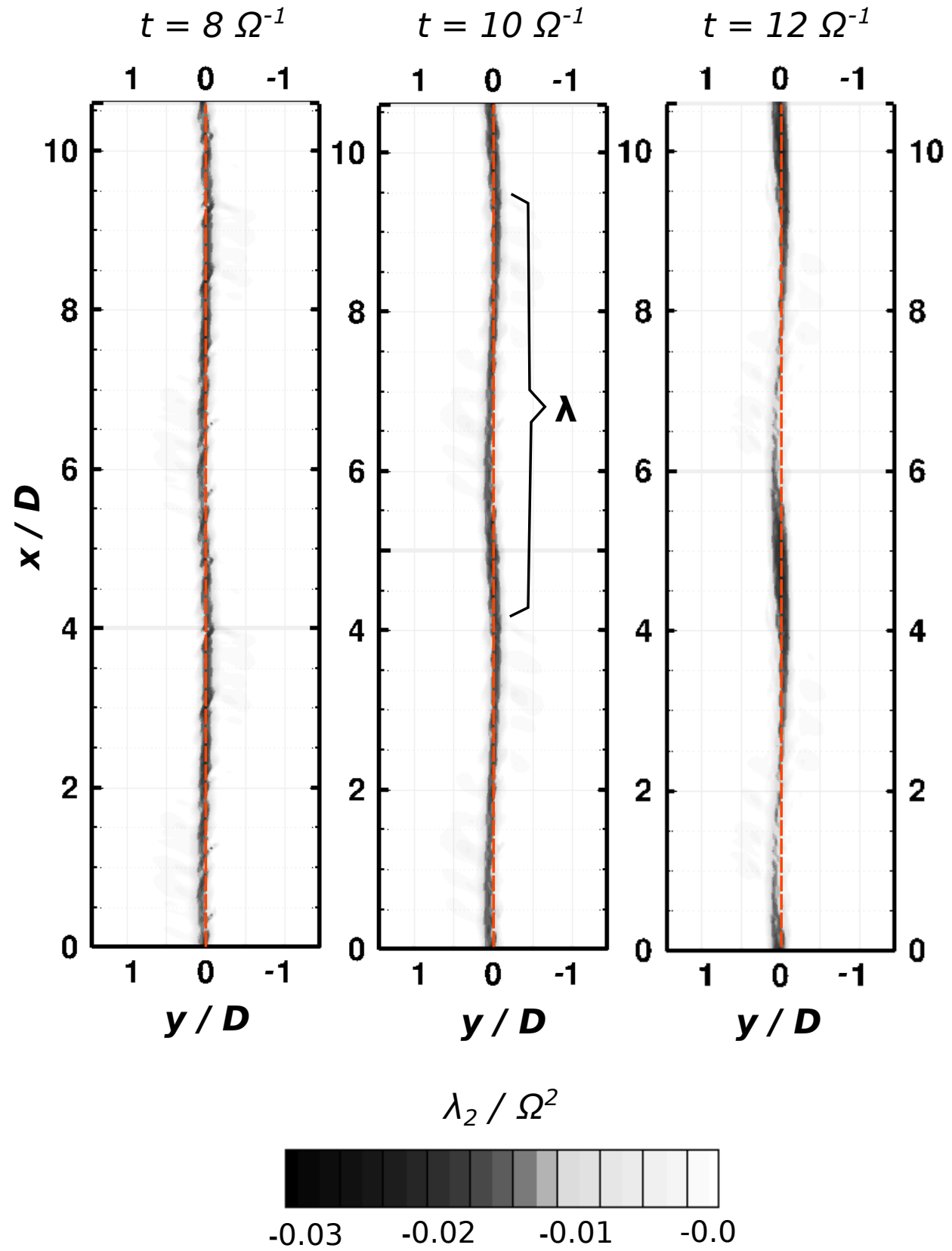

Figure 3. Shown are $\lambda_{2}$-values at center plane $z=0$ from times $8 \Omega^{-1}$ to $12 \Omega^{-1}$. A harmonic mode with wavelength $\lambda$ forms. The dashed red line indicates $y=0$.

increase of the $\widetilde{u}$ at the center plane is caused by the up- and downwards motions of the vortex and by the swirl of momentum towards the vortex center. The streamwise distribution of the maxima and minima of $\widetilde{u}$ are induced by the superimposed initial perturbation of the vortex with $\lambda=L_{x} / 2$. Regions with $\widetilde{u}$-extrema form on the $x y$-plane with a width $\approx D / 2$ and a length $\lambda / 2$. A circulation with anti-clockwise rotation around the $z$-axis can be induced in these regions (figure 5 ).

The evolving lateral shear $\partial_{y} \widetilde{u}$ in combination with a $\partial_{x} \widetilde{v}$ generates a vorticity component $\widetilde{\omega}_{z}$ shown in figure 6 . This vorticity corresponds to an anti-clockwise rotation 

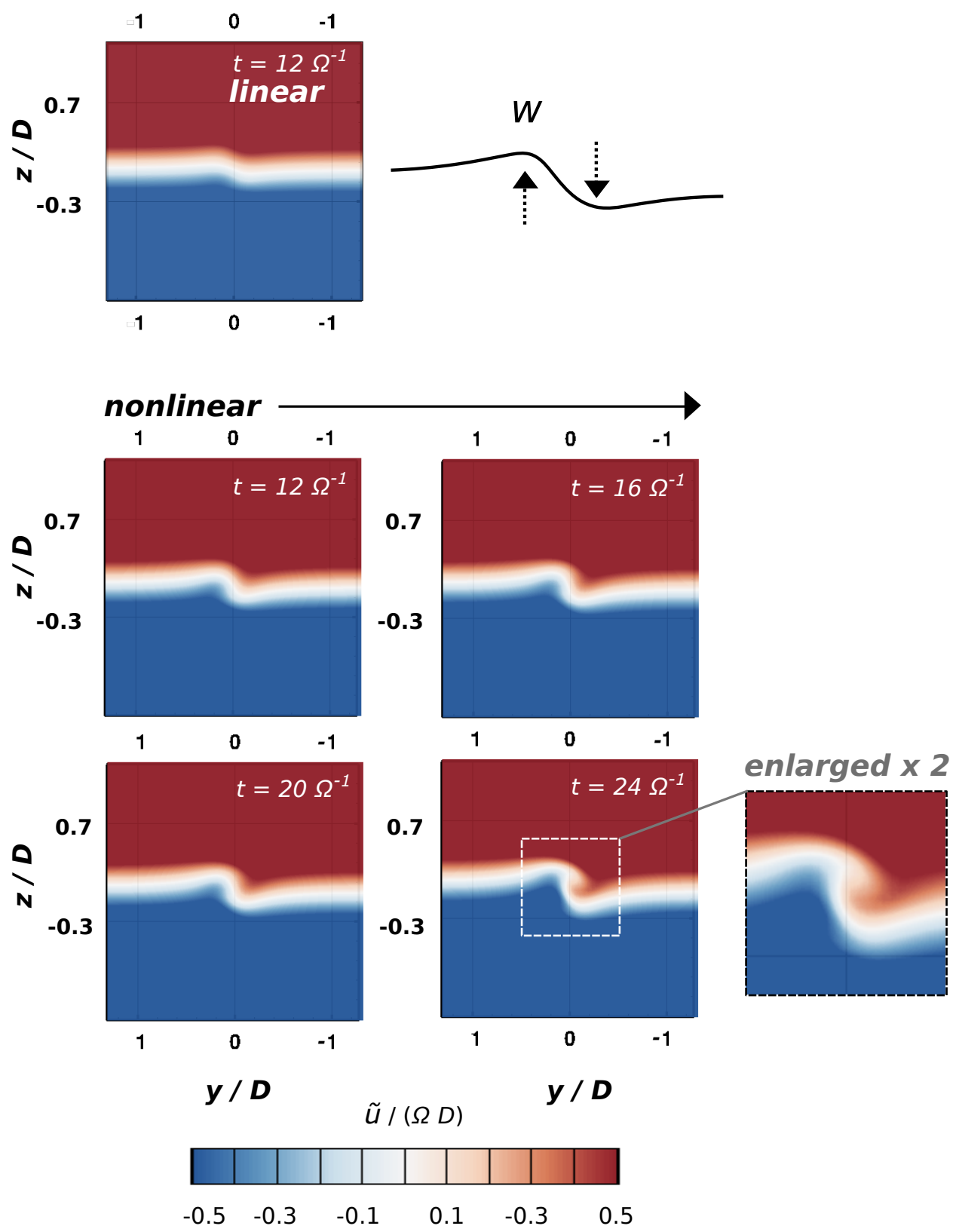

Figure 4. Streamwise velocity $\widetilde{u}(y, z)$ at center plane $x=L_{x} / 2$ at times $t=12 \Omega^{-1}$. The simulation with linearized advection equation (top left). The vertical velocity of the vortex $w$ transports the initially plane parallel shear $U(z)$ as sketched (top right) and produces a lateral gradient of the streamwise velocity $\partial_{y} \widetilde{u}<0$. The evolution with fully nonlinear advection is shown (below) for subsequent times $t=16 \Omega^{-1}$, $t=20 \Omega^{-1}$, and $t=24 \Omega^{-1}$. The nonlinear deformation of the shear in the plane is shown enlarged for $t=24 \Omega^{-1}$.

and leads to transverse deflections of the vortex seen either by the zero wind line in figure 5 or by the winding $\widetilde{\omega}_{z}$-contours in figure 6 . In time, the shear $\partial_{y} \widetilde{u}$ and the $\widetilde{\omega}_{z}$ in the $x y$-plane intensify. The transport mechanism is linear and indicated with a sketch in figure 4. Proportional to the background shear magnitude $\Omega$ and the vertical velocity of the vortex $w$, a change of streamwise momentum occurs in the form of a linear forcing 


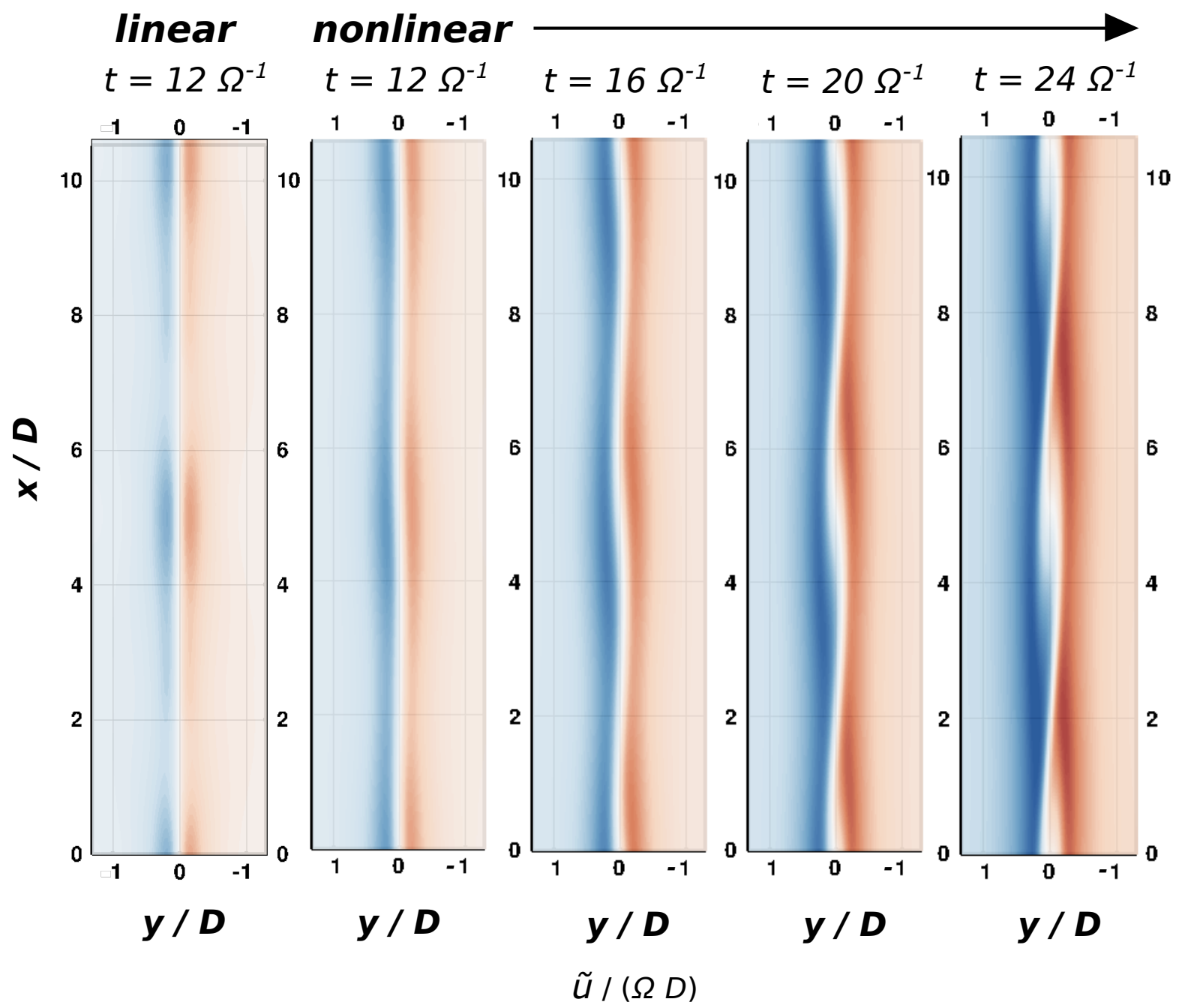

\section{linear}

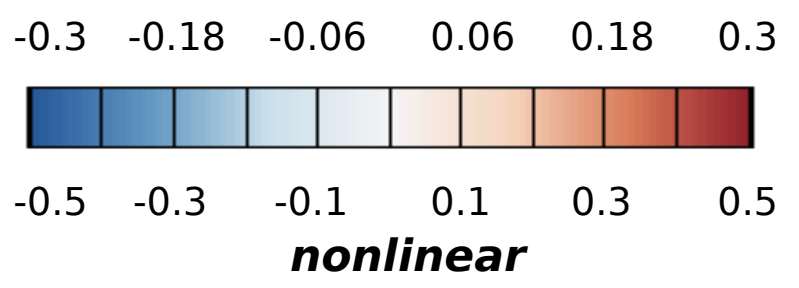

Figure 5. $\widetilde{u}(x, y)$ at center plane $z=0$ for the times from figure 4 .

$-w \Omega$. A laterally varying vertical velocity $w(y)$ creates a laterally varying streamwise momentum distribution. At the positions where $w(y)$ is locally high/low in the center plane, minima/maxima of horizontal momentum evolve.

At the early stage of the meandering motion these extrema of horizontal momentum are in phase in $x$-direction and remain spatially fixed in time for $t \lesssim 12 \Omega^{-1}$ as the $\lambda_{2^{-}}$ contours in figure 3. The time scale an air parcel needs to surround the region once, i.e. the eddy turnover time, is of order $\omega_{x}^{-1}$ and thus small compared to $\Omega^{-1}$. At later times $\gtrsim 16 \Omega^{-1}$ distinct $\widetilde{u}$-extrema form in the simulation as shear is transported by the vortex. This causes the minima/maxima to propagate in negative/positive streamwise 
$x$-direction with respect to the center plane. This advection occurs with streamwise velocity $\widetilde{u}$ in figure 5 .

So far, we have described the early stage excitation, where vorticity from the ambient shear is not yet wrapped in spirals around the wake vortex as in calculations by Kawahara et al. (1997). At later times greater than the eddy turnover time, a vortex spiral forms in the $y z$-plane as shown enlarged in figure 4. The formation of this spiral is due to a centrifugal instability of the vortex (Howard \& Gupta, 1962), as fluid is moving towards the center of the vortex and the pressure gradient no longer balances the centrifugal force.

\subsection{Nonlinear evolution}

The further evolution of the columnar vortex is characterized by three-dimensional dynamics. The vortex becomes twisted by its interaction with the strong lateral shear at a time of $20 \Omega^{-1}$ apparent with the deformed center line $\widetilde{u}=0$ in figure 5 . At $24 \Omega^{-1}$ a helical structure forms. This helix is sheared and periodic with a wavelength $\lambda$. This distance corresponds to the wavelength of the initial meandering mode. The vortex is now rotationally symmetric. The helical structure appears in the $\widetilde{\omega}_{z}$-field in the contour of the center plane as twisted structure with $\widetilde{\omega}_{z}>0$ in figure 6 with a width of $\approx D$. This periodicity of the helix in space with a long streamwise wavelength is furthermore apparent in the $\lambda_{2}$-isosurface at times $20 \Omega^{-1}$ and $24 \Omega^{-1}$ in figure 7 . The helix is advected by the streamwise shear flow $U(z)$.

Smaller scale dynamics acts upon the vortex. At $28 \Omega^{-1}$ the helical vortex appears to break up in several smaller columns. In the projection these smaller columns reveal to be twisted and staggered. Their formation is due to evolving vorticity $\Omega<0$ from $\partial_{z} \widetilde{u}<0$. Corresponding thinner lines of vorticity evolve when the vortex begins to form a spiral. This is apparent when a smaller vortex forms in the upper half of the initially present Lamb-Oseen vortex and overturns the shear a second time inside the LambOseen vortex. This further leads to a change in sign of $u$-momentum in $y$-direction at a scale of the diameter of the smaller vortex $\approx D / 4$. Gradually, this process leads to a meandering at smaller scales as apparent in the three dimensional $\lambda_{2}$-isosurfaces (figure 7).

\subsection{Transition to turbulence}

Small scale motion is apparent in the $\widetilde{u}$-velocity field as depicted in figure 4 at $24 \Omega^{-1}$. Turbulent scales distort the background shear layer $U(z)$ in a vertical layer with a thickness of about $D / 2$ around the center plane. The small-scale turbulence originates from the nonlinearity of the advection. In figure 7 the thinner vortex columns at $28 \Omega^{-1}$ originating from the meandering at smaller scales lead to smaller scale instabilities that evolve after $32 \Omega^{-1}$ to fine grained turbulence in the form of apparently incoherent small scale structures at the scale of the computational mesh. This small scale noise appears in the vicinity of the evanescent vortex at $36 \Omega^{-1}$. 


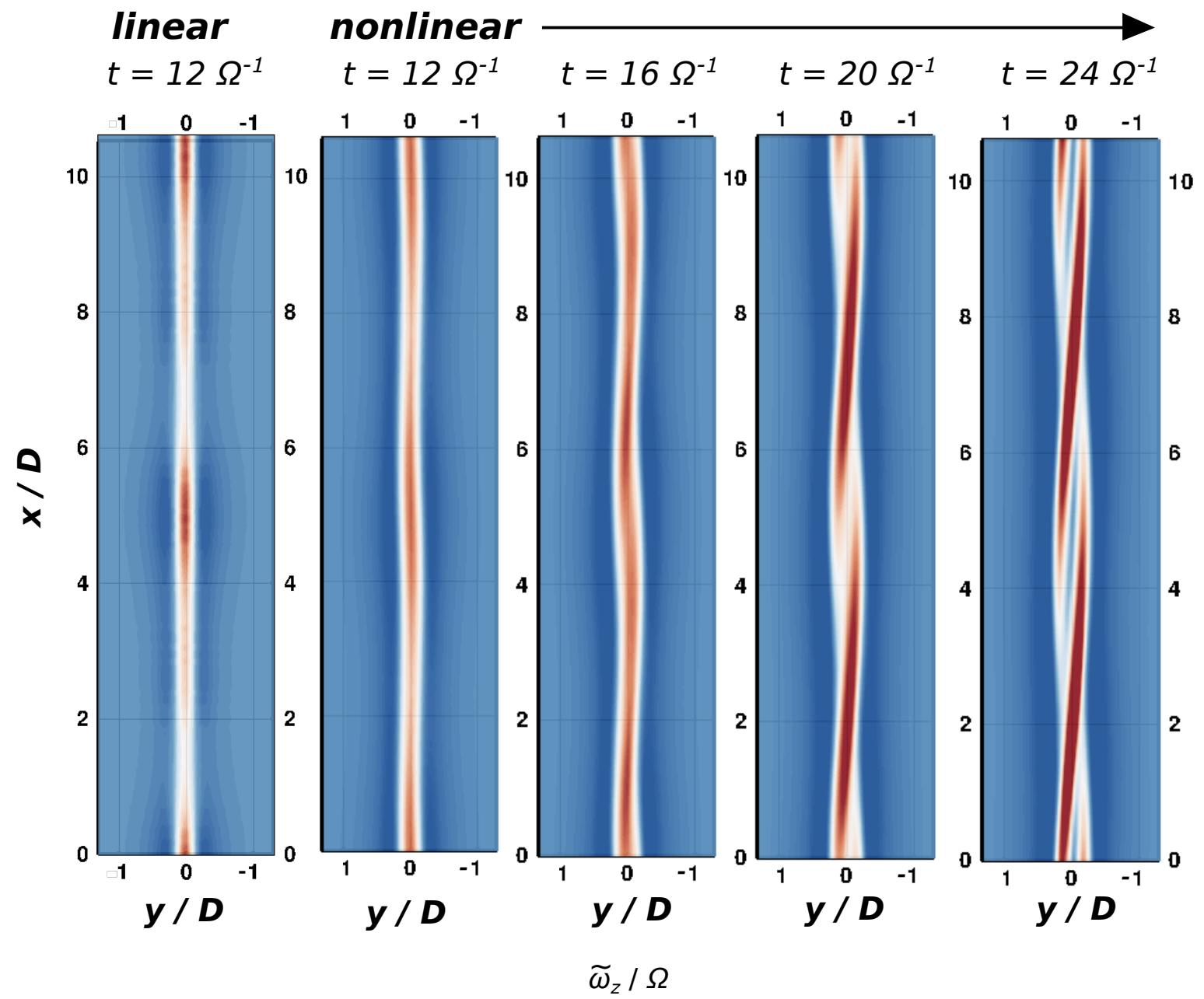

\section{linear}

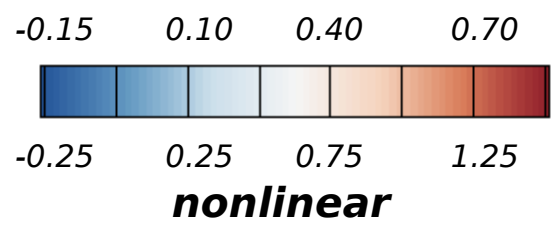

Figure 6. As figure 5 but for $\widetilde{\omega}_{z}$.

At later times we see a transition to fully turbulent flow as indicated by the spectrum of turbulent kinetic energy in figure 8. A local maximum occurs at a wavelength corresponding to the meandering wave. The inertial subrange shows a $k^{-5 / 3}$ slope. The flow does not transient to a turbulent state in the numerical simulations with linearized momentum equations. In the simulations with fully nonlinear equations the early stage excitation of the vortex meandering, the nonlinear evolution, and the transition to turbulence have been simulated. 


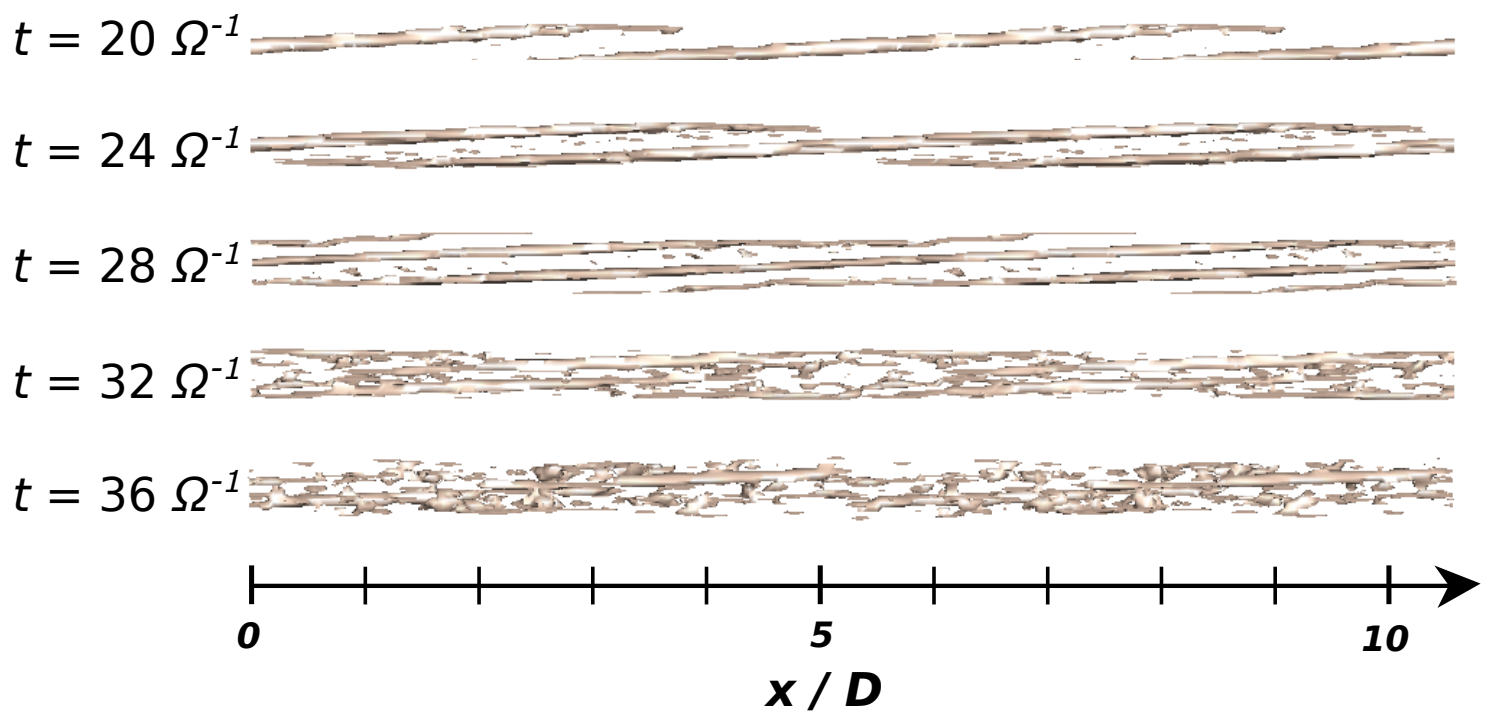

Figure 7. Three dimensional $\lambda_{2}$-isosurfaces are projected to a plane that is angulated by $\pi / 4$ to the center plane $z=0$. The vortex evolves from a time of $20 \Omega^{-1}$ to $36 \Omega^{-1}$.

\subsection{Additional sensitivity studies}

A variation of parameters in the simulation shows that the meandering excitation occurs similar for a wide range of $\omega_{0} \leq \Omega$ values. For larger $\omega_{0}$ values the shear forms a spiral and the described meandering occurs for a spectrum of scales simultaneously. For $\omega_{0} \ll \Omega$ values we found convergence of the flow towards the simulations with linear advection during the excitation phase of the vortex meandering by gradually decreasing the perturbation amplitude $a$ over a number of simulations. The diffusion of excited

wave modes in the simulations depends on SGS model. The effective eddy viscosity $\nu_{e d d y}$ is smaller in the implicit LES. There, the modes diffuse over a longer time period.

The simulations with flow perturbed by a set of random numbers in streamwise direction only, leads to a long streamwise wave of small amplitude in lateral and vertical direction. The simulations with random numbers varying in all three spatial directions lead to waves of smaller wavelength. These waves become unstable before the described meandering motion evolves. The random disturbances grow in amplitude at the top and bottom of the domain in the simulations with linear advection. The reason is a deviation from Galilean invariance of the simulated flow due to numerical discretization and forward-in-time differencing. The maximum/minimum streamwise advection velocities $U(z)$ occur at domain top/bottom $z= \pm L_{z} / 2$. There, the relatively high/low velocity leads to a higher growth of the initial perturbations than at the domain center plane. This effect is not seen in the simulations with fully nonlinear advection.

\section{Discussion}

Our theoretical analysis and numerical simulations reveal an instability of a wind turbine far-wake vortex in strong shear flow. This instability occurs when the vorticity of the 
vortex interacts linearly with the background shear. This interaction excites and sustains the vortex meandering in the form of a long streamwise wave on the vortex. This wave undulates the wake vortex in lateral direction. The linear stability analysis reveals a time scale $\Omega^{-1}$ for the growth rate of this mode. Numerical simulations confirm the growth of a vorticity component forming perpendicular to vortex column $\boldsymbol{\omega}$ and background shear $\Omega$. This vorticity undulates the vortex in lateral direction. Long streamwise waves evolve on the vortex with wavelength $\lambda$ and the vortex forms a helix. Over a time scale of the vortex turnover time a spiral forms in the $y z$-plane and fluid moves towards the vortex center in the $y z$-plane. The helix persists and its fully nonlinear evolution is simulated until instabilities evolve at smaller scales $\ll D$. Due to these smaller scale instabilities the flow transients to a turbulent state. Finally, the vortex diffuses by turbulent mixing.

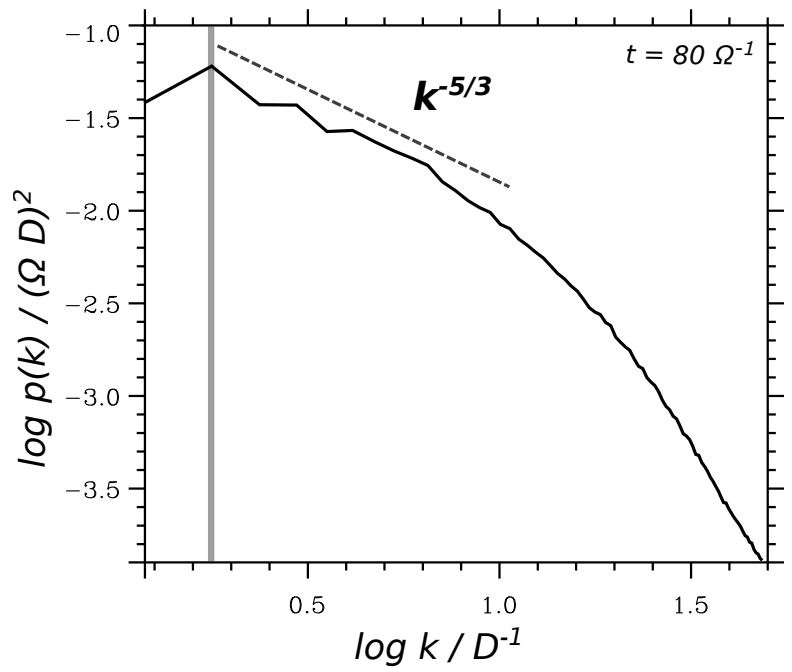

Figure 8. The vertically and laterally averaged power spectrum from a Fourier analysis in $x$-direction of the resolved turbulent kinetic energy shows a $k^{-5 / 3}$ slope after a time of $80 \Omega^{-1}$. A wave mode $\lambda$ is apparent at a wave number $\log k / D^{-1} \approx 0.25$ (vertical grey line). This wave number corresponds to a length scale of $3.5 D \approx \lambda / 2$.

The far-wake vortex meandering instability is now compared to the instability of aircraft wake vortices studied by Crow (1970): On the aircraft wake vortex pair streamwise waves form along the vortex column that become unstable. These waves are excited by mutual induction of the parallel columnar vortices. In our study the wave is excited due to an interaction of background shear perpendicular to one vortex, i.e. $\boldsymbol{\omega} \perp \boldsymbol{\Omega}$. The wave evolves in streamwise direction and over time as for the Crow instability. We use neutral background stratification as in the stability analysis of a wind turbine wake by Iungo et al. (2013) and of the wake vortex pair by Crow (1970). The vortex vorticity is initially distributed uniformly across the vortex diameter, as for each wake vortex in the configuration by Crow (1970). In addition, the far-wake vortex may stretch or contract radially depending on downstream position. Thereby, the vorticity changes in accordance with the radius to preserve the circulation. In 
general, the simulated instability is only part of the solution. In our theory different geometries are possible for the excited modes as for the aircraft wake vortices (Crow, 1970, states this in his chapter 3). When the components of the lateral and vertical vorticity amplitudes are both real/imaginary, then the wave oscillates in a plane. If these amplitudes differ by a factor $i$, then a helix evolves. Different to the instability found by Crow (1970), there exists no preferred wave length for the meandering instability. Only an indirect selection of wave numbers exists, as the meandering instability becomes more pronounced for longer wave lengths, when advection and diffusion become negligible.

For one isolated vortex tube with strong vorticity, Arendt \& Fritts (1998) studied the effect of weak background shear by means of linear stability analysis. Besides the shear, a straining flow acted on the vortex tube. Arendt \& Fritts (1998) found an instability in the form of a sinusoidal perturbation of the vortex in a plane at an angle of $-\pi / 4$. In the absence of the straining flow the vortex is also unstable. Different to our analysis, the instability occurs in weak background shear and for short wavelengths.

Our findings do not exclude that meandering may be caused by advection of the wake by coherent structures larger than the turbine diameter, as taken into account by Trujillo et al. (2011) and Larsen et al. (2008). Okulov et al. (2014) suggest interaction of the far wake with large three dimensional vortices from bluff body vortex shedding at the turbine as possible explanation for the far-wake meandering. As Iungo et al. (2013) showed, unstable modes in the wind turbine wake can also occur when the inflow velocity is uniform across the height of the turbine. Our results emphasize the significance of strong background shear as one further cause for the meandering of wind turbine farwake vortices.

\section{Conclusion}

This paper constitutes an alternative explanation for the excitation of wake vortex meandering behind wind turbines. Linear stability analysis and numerical simulations convey that strong homogenous shear linearly excites and sustains vortex meandering. In the numerical simulations the linearly excited long wave mode amplifies and persists in coexistence with smaller scales instabilities throughout the nonlinear simulations. The meandering is sustained by interaction between strong external shear with weak wake rotation until the flow transients to a fully turbulent state.

\section{Acknowledgements}

The author JS thanks N. Harnik (Tel Aviv University) and O. M. Umurhan (University of California) for fruitful discussions during the $5^{\text {th }}$ international conference on 'Bifurcations and Instabilities in Fluid Dynamics' in Haifa, Israel in 2013. We appreciate the constructive comments by our colleague F. Holzäpfel (DLR) and two anonymous referees on an earlier version of the manuscript. JS wants to thank G. Craig (University of Munich) for constructive advice on the linear theory. JS linearized the advection 
equations for the flow configuration in our geophysical flow solver EULAG after a fruitful exchange with P.K. Smolarkiewicz (ECMWF). Computational resources have been made available by the German Climate Computing Center (DKRZ) through support from the German Federal Ministry of Education and Research (BMBF).

\section{References}

Arendt, S. \& Fritts, D. C. 1998 The instability of a vortex tube in a weak external shear and strain. Phys. Fluids 10, 530-532.

Banta, R. M., Pichugina, Y. L., Kelley, N. D., Hardesty, R. M. \& Brewer, W. A. 2013 Wind energy meteorology: Insight into wind properties in the turbinerotor layer of the atmosphere from high-resolution doppler lidar. Bull. Amer. Meteorol. Soc. 94, 883-902.

Chamorro, L. P. \& Porté-Agel, F. 2010 Effects of thermal stability and incoming boundary-layer flow characteristics on wind-turbine wakes: A wind-tunnel study. Bound. Layer Meteorol. 136, 515-533.

Crow, S. C. 1970 Stability theory for a pair of trailing vortices. AIAA J. 8, 2172-2179.

Glauert, H. 1926 The elements of aerofoil and airscrew theory. Cambridge University Press.

Grinstein, F. F., Margolin, L. G. \& Rider, W. J. 2007 Implicit large eddy simulation: computing turbulent fluid dynamics. Cambridge University Press.

Hirth, B. D., Schroeder, J. L., Gunter, W. S. \& Guynes, J. G. 2012 Measuring a utility-scale turbine wake using the TTUKa mobile research radars. J. Atmos. Oceanic Technol. 29, 765-771.

Howard, L. N. \& Gupta, A. 1962 On the hydrodynamic and hydromagnetic stability of swirling flows. J. Fluid Mech. 14, 463-476.

Iungo, G., Viola, F., Camarri, S., Porté-Agel, F. \& Gallaire, F. 2013 Linear stability analysis of wind turbine wakes performed on wind tunnel measurements. $J$. Fluid Mech. 737, 499-526.

Ivanell, S., Mikkelsen, R., Sørensen, J. N. \& Henningson, D. 2010 Stability analysis of the tip vortices of a wind turbine. Wind Energy 13, 705-715.

Jeong, J., Hussain, F., Schoppa, W. \& Kim, J. 1997 Coherent structures near the wall in a turbulent channel flow. J. Fluid Mech. 332, 185-214.

KäsleR, Y. 2011 Doppler-Windlidar-Messungen der Umströmung einer Windenergieanlage. PhD thesis, Carl von Ossietzky Univ. Oldenburg.

Käsler, Y., Rahm, S., Simmet, R. \& Kühn, M. 2010 Wake measurements of a multi-mw wind turbine with coherent long-range pulsed doppler wind lidar. J. Atmos. Oceanic Technol. 27, 1529-1532. 
Kawahara, G., Kida, S., Tanaka, M. \& Yanase, S. 1997 Wrap, tilt and stretch of vorticity lines around a strong thin straight vortex tube in a simple shear flow. $J$. Fluid Mech. 353, 115-162.

Larsen, G. C., Madsen, H. A., Thomsen, K. \& Larsen, T. J. 2008 Wake meandering: a pragmatic approach. Wind Energy 11, 377-395.

Margolin, L., Smolarkiewicz, P. \& Sorbjan, Z. 1999 Large-eddy simulations of convective boundary layers using nonoscillatory differencing. Physica D 133, 390-397.

Medici, D. \& Alfredsson, P. 2006 Measurements on a wind turbine wake: 3D effects and bluff body vortex shedding. Wind Energy 9, 219-236.

Meyers, J. \& Meneveau, C. 2013 Flow visualization using momentum and energy transport tubes and applications to turbulent flow in wind farms. J. Fluid Mech. 715, $335-358$.

Okulov, V. \& Sorensen, J. 2007 Stability of helical tip vortices in a rotor far wake. J. Fluid Mech. 576, 1-25.

Okulov, V. L., Naumov, I. V., Mikkelsen, R. F., Kabardin, I. K. \& SøREnsen, J. N. 2014 A regular strouhal number for large-scale instability in the far wake of a rotor. J. Fluid Mech. 747, 369-380.

Prusa, J., Smolarkiewicz, P. \& Wyszogrodzki, A. 2008 EUlAG, a computational model for multiscale flows. Comput. \& Fluids 37, 1193-1207.

Saffman, P. G. 1992 Vortex dynamics. Cambridge University Press.

Schumann, U. 1975 Subgrid scale model for finite difference simulations of turbulent flows in plane channels and annuli. J. Comput. Phys. 18, 376-404.

Smolarkiewicz, P. K. \& Charbonneau, P. 2013 EUlAG, a computational model for multiscale flows: An MHD extension. J. Comput. Phys. 236, 608-623.

Smolarkiewicz, P. K., Kühnlein, C. \& Wedi, N. P. 2014 A consistent framework for discrete integrations of soundproof and compressible PDEs of atmospheric dynamics. J. Comput. Phys. 263, 185-205.

Sørensen, J. N. 2011 Aerodynamic aspects of wind energy conversion. Annu. Rev. Fluid Mech. 43, 427-448.

Trujillo, J.-J., Bingöl, F., Larsen, G. C., Mann, J. \& Kühn, M. 2011 Light detection and ranging measurements of wake dynamics. Part II: Two-dimensional scanning. Wind Energy 14, 61-75.

VALLIS, G. 2006 Atmospheric and oceanic fluid dynamics: fundamentals and large-scale circulation. Cambridge University Press. 